SNUTP01-042

\title{
Supersymmetry-based Approach to Quantum Particle Dynamics on a Curved Surface with Non-zero Magnetic Field
}

\author{
Seok Kim円 and Choonkyu Lee' \\ Department of Physics and Center for Theoretical Physics \\ Seoul National University, Seoul 151-742, Korea
}

\begin{abstract}
We present the $N=2$ supersymmetric formulation for the classical and quantum dynamics of a nonrelativistic charged paritcle on a curved surface in the presence of a perpendicular magnetic field. For a particle moving on a constant-curvature surface in a constant magnetic field, our Hamiltonian possesses the shape-invariance property in addition. On the surface of a sphere and also on the hyperbolic plane, we exploit the supersymmetry and shape-invariance properties to obtain complete solutions to the corresponding quantum mechanical problems.
\end{abstract}

*Email address: calaf2@snu.ac.kr

†Email address: cklee@phya.snu.ac.kr 


\section{INTRODUCTION}

The Landau levels and associated wavefunctions [1] are of crucial importance in understanding many striking observations made for two-dimensional or planar systems of electrons in a perpendicular magnetic field, as in the cases of the de Haas-van Alphen effect in metals [2] and the quantum Hall effect[3,4]. To explain the quantum Hall effect in particular, Laughlin [5] in his ground-breaking effort proposed variational wave functions describing incompressible states corresponding to special rational filling fractions of the highly degenerate lowest Landau level. Haldane [6] then introduced a variant of the Laughlin wave functions by considering a system of electrons constrained to the surface of a sphere. On the two-sphere, the fact that single-particle energy levels have finite degeneracy only makes numerical simulations more tractable and allows for a simplified treatment of the thermodynamic limit. Note that the one-particle states for the latter case can also be found in closed forms.

It is the supersymmetry that is largely responsible for the mathematical structure of the Landau levels. This is seen by considering the Pauli Hamiltonian for a nonrelativistic spin-1/2 particle in a plane,

$$
\begin{aligned}
H & =\frac{1}{2 m}[\vec{p}-\vec{A}(\vec{x})]^{2}-\frac{1}{2 m} \sigma_{3} B(\vec{x}) \\
& =\frac{1}{2 m}\left[\sigma_{1}\left(p_{1}-A_{1}(\vec{x})\right)+\sigma_{2}\left(p_{2}-A_{2}(\vec{x})\right)\right]^{2},
\end{aligned}
$$

where $\vec{x} \equiv\left(x^{1}, x^{2}\right), B(\vec{x})=\partial_{1} A_{2}(\vec{x})-\partial_{2} A_{1}(\vec{x})$, and $\left(\sigma_{1}, \sigma_{2}, \sigma_{3}\right)$ are the usual Pauli matrices. This quantum system is known to possess an $N=2$ supersymmetry[7,8], and one may exploit this supersymmetry to find for instance the exact zero-energy ground-state wavefunctions of the system in the presence of an arbitrary, spatially dependent, magnetic field $[9,10]$. In this paper we shall generalize the $N=2$ supersymmetry property of the Pauli Hamiltonian to the case when the underlying two-dimensional manifold is a curved surface. This system can also be recast as an $N=2$ supersymmetric system involving a pair of Schrödinger Hamiltonians (i.e., for spinless particles) defined on the curved surface.

For a complete quantum-mechanical solution, however, the existence of supersymmetry is not enough — we need also the shape-invariance[11,12]. For the Pauli system defined on 
a curved surface, it turns out that shape-invariant Hamiltonians are obtained when both the scalar curvature and the external magnetic field (perpendicular to the surface) are restricted to be constant. The system defined on the two-sphere or on the hyperbolic plane(also called the pseudo-sphere) belongs to such, and these cases provide interesting generalizations of the planar Landau level problem. In this paper we thus find complete energy eigenfunctions of the corresponding quantum system by exploiting supersymmetry and shape-invariance. See Refs.[13-15] for some previous works on these problems, but with different emphasis. The purpose of the present work is to provide a concise, yet self-contained, treatment of the whole problem from the perspective of supersymmetry. A particularly comprehensive treatment using more traditional method of mathematical physics is given in Ref. [14]. [Very recently, there appeared also a preprint by G.A.Mezincescu and L.Mezincescu [16] which contains materials related to the present work].

This paper is organized as follows. In Section II we begin with the Lagrangian/Hamiltonian description of the classical $N=2$ supersymmetric model of a nonrelativistic spinning particle coupled to an external gauge field in a general two-dimensional manifold. The corresponding quantum theory is discussed in Section III. In the Schrödinger

picture, we here find the matrix Schrödinger equation which may be recognized either as a natural curved-space generalization of the usual Pauli equation for a spin-1/2 particle, or as that appropriate to a system consisting of a pair of superpartner Hamiltonians for a spinless particle. In Section IV we study the cases described by shape-invariant Hamiltonians, that is, the Landau Hamiltonian on the two-sphere or on the hyperbolic plane. For both cases, the energy levels and complete energy eigenfunctions are produced by our supersymmetry-based approach. Concluding remarks are made in Section V.

\section{CLASSICAL THEORY WITH N=2 SUPERSYMMETRY}

In this section we will discuss the classical theory of a nonrelativistic charged spinning particle on a two-dimensional curved surface with the metric $g_{\mu \nu}(x)$ for conveniently 
chosen coordinates $x^{\mu}=\left(x^{1}, x^{2}\right)$. Interaction with external vector potentials $A_{\mu}(x) \equiv$ $\left(A_{1}(x), A_{2}(x)\right)$ is included. To describe a spinning particle, it is useful to introduce the zweibein $e_{a}^{\mu}(x)$ ( $a=1,2$ refer to components relative to the local orthonormal frame on the surface) satisfying

$$
e_{\mu}^{a}(x) e_{b}^{\mu}(x)=\delta_{b}^{a}, \quad e_{\mu}^{a}(x) e_{\nu}^{a}(x)=g_{\mu \nu}(x),
$$

and the corresponding spin-connection $\omega_{\mu a b}(x) \equiv \omega_{\mu}(x) \epsilon_{a b}$ which is related to the zweibeins in the usual manner [17.

On a curved surface, electromagnetic potentials are described by a one-form $\mathbf{A}=A_{\mu} d x^{\mu}$ and are used to define the field strength two-form by $\mathbf{F}=d \mathbf{A}=\frac{1}{2} F_{\mu \nu} d x^{\mu} \wedge d x^{\nu}$ where $F_{\mu \nu}=\partial_{\mu} A_{\nu}-\partial_{\nu} A_{\mu}$. Using the frame one-form $\mathbf{e}^{a}=e_{\mu}^{a}(x) d x^{\mu}$, the electromagnetic field strength is equivalently expressed as $\mathbf{F}=B(x) \mathbf{e}^{1} \wedge \mathbf{e}^{2}$ with $B(x)=\frac{1}{2} \epsilon_{a b} e_{\mu}^{a}(x) e_{\nu}^{b}(x) F^{\mu \nu}(x), \epsilon_{a b}$ being a totally-skew symbol. The quantity $B(x)$ can be taken to describe the strength of the magnetic field in the direction 'normal' to the surface; this is natural since one may represent the magnetic flux (or the first Chern number) over a surface $M$ by $\Phi=\int_{M} \mathbf{F}=\int_{M} B d($ vol $)$ with the volume form $d(v o l)=\sqrt{g} d x^{1} \wedge d x^{2}=\mathbf{e}^{1} \wedge \mathbf{e}^{2}$, where $g=\operatorname{det}\left(g_{\mu \nu}\right)$.

Then, taking as our dynamical variables two bosonic position coordinates $x^{\mu}(t)$ and two real Grassmann variables (for the spin degrees of freedom [18]) $\psi_{a}(t)$, consider the Lagrangian 19

$$
\begin{aligned}
L(x, \dot{x}, \psi, \dot{\psi}) & =\frac{m}{2} g_{\mu \nu}(x) \dot{x}^{\mu} \dot{x}^{\nu}+\frac{i}{2} \psi_{a} \dot{\psi}_{a}+\dot{x}^{\mu} A_{\mu}(x) \\
& -\dot{x}^{\mu} \omega_{\mu}(x) S(\psi)+\frac{1}{m} B(x) S(\psi),
\end{aligned}
$$

where $S(\psi) \equiv-\frac{i}{2} \epsilon_{a b} \psi_{a} \psi_{b}$ represents the spin of the particle. Brief explanations on various terms appearing in the Lagrangian (2.2) might be desirable. The first and third terms are the ones needed to describe a spinless particle moving on a curved surface under the action of external vector potentials. The second and fourth terms are responsible for the spin dynamics on a curved surface ; in fact, these two terms may be combined to yield the expression with the covariant derivative $\frac{D}{d t}[20]$ : 


$$
\frac{i}{2} \psi_{a}\left(\dot{\psi}_{a}+\dot{x}^{\mu} \omega_{\mu a b}(x) \psi_{b}\right) \equiv \frac{i}{2} \psi_{a} \frac{D}{d t} \psi_{a}
$$

The last term $B(x) S(\psi)$, which represents the interaction between the spin and external magnetic field with a very specific gyromagnetic ratio, has been included to make the above theory be invariant under the supersymmetry transformation

$$
\begin{gathered}
\delta x^{\mu}=i \frac{\epsilon}{\sqrt{m}} e_{a}^{\mu}(x) \psi_{a} \equiv i \frac{\epsilon}{\sqrt{m}} \psi^{\mu}, \\
\delta \psi^{\mu}=-\epsilon \sqrt{m} \dot{x}^{\mu}
\end{gathered}
$$

with a real Grassman parameter $\epsilon$. Note that, using $\psi_{a},(2.4 \mathrm{~b})$ can also be written as

$$
\delta \psi_{a}=-\frac{\epsilon}{\sqrt{m}} e_{\mu a}(x)\left(m \dot{x}^{\mu}-\omega^{\mu}(x) S(\psi)\right) .
$$

The Lagrangian invariant under the transformation $(2.4 \mathrm{a}, \mathrm{b})$ exists in general spatial dimension, but, for our Lagrangian (2.2) which applies specifically to the two-dimensional case, we have in fact an additional supersymmetry. Such extended supersymmetry structure can be seen most clearly in the Hamiltonian formulation. By the simple Legendre transform with (2.2), the Hamiltonian appropriate to our system reads

$$
H=\frac{1}{2 m} g^{\mu \nu}(x)\left[p_{\mu}-A_{\mu}(x)+\omega_{\mu}(x) S(\psi)\right]\left[p_{\nu}-A_{\nu}(x)+\omega_{\nu}(x) S(\psi)\right]-\frac{1}{m} B(x) S(\psi)
$$

with the Poisson brackets

$$
\left\{x^{\mu}, p_{\nu}\right\}_{P B}=\delta_{\nu}^{\mu},\left\{\psi_{a}, \psi_{b}\right\}_{P B}=-i \delta_{a b},\left\{x^{\mu}, \psi_{a}\right\}_{P B}=0
$$

Here, following Ref. [18], the Poisson brackets of two dynamical variables $A(x, p, \psi)$ and $B(x, p, \psi)$ are defined by

$$
\{A, B\}_{P B} \equiv \frac{\partial A}{\partial x^{\mu}} \frac{\partial B}{\partial p_{\mu}}-\frac{\partial A}{\partial p_{\mu}} \frac{\partial B}{\partial x^{\mu}}-i A \frac{\overleftarrow{\partial}}{\partial \psi_{a}} \frac{\vec{\partial}}{\partial \psi_{a}} B
$$

Now one can easily verify that the above supersymmetry transformation, which can equivalently be written as

$$
\delta x^{\mu}=i \frac{\epsilon}{\sqrt{m}} e_{a}^{\mu}(x) \psi_{a}, \quad \delta \psi_{a}=-\frac{\epsilon}{\sqrt{m}} e_{a}^{\mu}(x)\left(p_{\mu}-A_{\mu}(x)\right)
$$


is generated by the supercharge $Q_{1} \equiv \frac{i}{\sqrt{m}} \psi_{a} e_{a}^{\mu}(x)\left(p_{\mu}-A_{\mu}(x)\right)$, i.e.,

$$
\delta x^{\mu}=\left\{x^{\mu}, \epsilon Q_{1}\right\}_{P B}, \delta \psi_{a}=\left\{\psi_{a}, \epsilon Q_{1}\right\}_{P B} .
$$

Note that $\left\{H, Q_{1}\right\}_{P B}=0$, and hence the charge $Q_{1}$ is conserved. Also the Hamiltonian (2.6) can be expressed as

$$
H=-\frac{i}{2}\left\{Q_{1}, Q_{1}\right\}_{P B}
$$

Now, to exhibit the $N=2$ supersymmetry for our system, define

$$
\psi \equiv \frac{1}{\sqrt{2}}\left(\psi_{1}-i \psi_{2}\right), e^{\mu}(x) \equiv \frac{1}{\sqrt{2}}\left(e_{1}^{\mu}(x)-i e_{2}^{\mu}(x)\right)
$$

where the subscript 1,2 refer to components relative to the local orthonormal frame. Also let us denote the complex conjugates of $\psi, e^{\mu}(x)$ by $\bar{\psi}, \bar{e}^{\mu}(x)$. Then it is shown by direct calculations that a pair of complex supercharges

$$
Q \equiv \frac{i}{\sqrt{m}} \psi \bar{e}^{\mu}(x)\left(p_{\mu}-A_{\mu}(x)\right), \bar{Q} \equiv-\frac{i}{\sqrt{m}} \bar{\psi} e^{\mu}(x)\left(p_{\mu}-A_{\mu}(x)\right)
$$

satisfy the following $N=2$ supersymmetry algebra:

$$
\begin{gathered}
\{Q, Q\}_{P B}=\{\bar{Q}, \bar{Q}\}_{P B}=0 \\
i\{Q, \bar{Q}\}_{P B}=H, \\
\{H, Q\}_{P B}=\{H, \bar{Q}\}_{P B}=0 .
\end{gathered}
$$

Note that $Q_{1}=(Q-\bar{Q})$, and $(2.14 \mathrm{~b})$ is just a rewriting of (2.11).

For a general dynamical variable $A$, the $N=2$ supersymmetry transformation can be represented by $\delta A=\{A, \varepsilon Q+\bar{Q} \bar{\varepsilon}\}_{P B}$, where $\varepsilon$ is a complex Grassmannian parameter. Especially, for $x^{\mu}$ and $\psi$, the transformation reads

$$
\delta x^{\mu}=\frac{i}{\sqrt{m}}\left(\varepsilon \psi \bar{e}^{\mu}(x)+\bar{\varepsilon} \bar{\psi} e^{\mu}(x)\right), \delta \psi=-\frac{1}{\sqrt{m}} \bar{\varepsilon} e^{\mu}\left(p_{\mu}-A_{\mu}(x)\right) .
$$

This reduces to (2.9) if $\varepsilon$ is taken to be real Grassmannian (i.e., $\varepsilon=\epsilon$ ). With $\varepsilon=i \epsilon$, on the other hand, we obtain from (2.15) the second supersymmetry transformation 


$$
\delta x^{\mu}=\frac{i \epsilon}{\sqrt{m}} \epsilon_{a b} e_{a}^{\mu}(x) \psi_{b}, \delta \psi_{a}=\frac{\epsilon}{\sqrt{m}} \epsilon_{a b} e_{b}^{\mu}(x)\left(p_{\mu}-A_{\mu}(x)\right) .
$$

We remark that, for the Lagrangian (2.2), this second supersymmetry can be described by the transformation (cf.(2.4a) and (2.5))

$$
\delta x^{\mu}=\frac{i \epsilon}{\sqrt{m}} \epsilon_{a b} e_{a}^{\mu}(x) \psi_{b}, \delta \psi_{a}=\frac{\epsilon}{\sqrt{m}} \epsilon_{a b} e_{\mu b}(x)\left(m \dot{x}^{\mu}-\omega^{\mu}(x) S(\psi)\right)
$$

For $\psi^{\mu}=e_{a}^{\mu}(x) \psi_{a}$, this leads to the transformation $\delta \psi^{\mu}=\frac{\epsilon}{\sqrt{m}}\left(\epsilon_{\nu}^{\mu}(x) m \dot{x}^{\nu}-\right.$ $\left.\Gamma_{\nu \rho}^{\mu}(x) g^{\nu \rho}(x) S(\psi)\right)$, where $\epsilon_{\nu}^{\mu}(x) \equiv \epsilon_{a b} e_{a}^{\mu}(x) e_{\nu b}(x)$ and $\Gamma_{\nu \rho}^{\mu}(x)$ is the Christoffel symbol. [Note that $\Gamma_{\nu \rho}^{\mu}=\frac{1}{2} g^{\mu \lambda}\left(\partial_{\nu} g_{\rho \lambda}+\partial_{\rho} g_{\nu \lambda}-\partial_{\lambda} g_{\nu \rho}\right)$ and $\omega_{\mu a b}(x) e_{b}^{\nu}(x)=-\partial_{\mu} e_{a}^{\nu}(x)-\Gamma_{\mu \rho}^{\nu}(x) e_{a}^{\rho}(x)$.]

\section{QUANTUM THEORY WITH N=2 SUPERSYMMETRY}

When the classical Hamiltonian is given as in (2.6), the purpose of this section is to set up the corresponding quantum theory, with the $N=2$ supersymmetry realized by appropriate Hilbert-space operators. In the Schrödinger picture, basic dynamical variables, i.e., $x^{\mu}, p_{\nu}$ and $\psi_{a}$ become Hermitian operators satisfying the algebraic relations

$$
\begin{aligned}
& {\left[x^{\mu}, p_{\nu}\right]=i \delta_{\nu}^{\mu},\left\{\psi_{a}, \psi_{b}\right\}=\delta_{a b},} \\
& {\left[x^{\mu}, x^{\nu}\right]=\left[p_{\mu}, p_{\nu}\right]=\left[x^{\mu}, \psi_{a}\right]=\left[p_{\mu}, \psi_{a}\right]=0,}
\end{aligned}
$$

where the brackets [ ] and \{\} denote commutator and anticommutator, respectively. Note

that if the non-Hermitian operator $\psi=\frac{1}{\sqrt{2}}\left(\psi_{1}-i \psi_{2}\right)$ and its conjugate $\bar{\psi}$ are used, the relations $\left\{\psi_{a}, \psi_{b}\right\}=\delta_{a b}$ are equivalent to the followings: $\{\psi, \bar{\psi}\}=1$ and $\psi^{2}=\bar{\psi}^{2}=0$. In order to secure the desired supersymmetric system, we must here require that the quantum Hamiltonian operator be expressed as

$$
H=\{Q, \bar{Q}\}
$$

with a suitably chosen supercharge operator $Q$ (which is of course related to the corresponding classical expression in (2.13)). Hence, in our discussions below, we are going to pay much attention to the construction of the supercharge operators. 
To find the quantum operators corresponding to the classical expressions (2.13), one will have to settle first the operator ordering problem concerning $p_{\mu}, e^{\mu}(x)$ and $\bar{e}^{\mu}(x)$. Following Ref.[21], we will adopt the Weyl-ordered form, which in the present case yields the operator

$$
\begin{aligned}
Q & =\frac{i}{\sqrt{m}} \psi\left[\frac{1}{2}\left\{\bar{e}^{\mu}(x), p_{\mu}\right\}-\bar{e}^{\mu}(x) A_{\mu}(x)\right] \\
& =\frac{i}{\sqrt{m}} \psi \bar{e}^{\mu}(x)\left[p_{\mu}-A_{\mu}(x)+\frac{1}{2} \omega_{\mu}(x)+\frac{i}{2} \Gamma_{\nu \mu}^{\nu}(x)\right]
\end{aligned}
$$

Here, to obtain the second form, we have assumed that $p_{\mu}$ has the usual differential operator realization, i.e., $p_{\mu}=-i \partial_{\mu}$, and used the relation $\partial_{\mu} \bar{e}^{\nu}(x)=i \omega_{\mu}(x) \bar{e}^{\nu}(x)-\Gamma_{\mu \rho}^{\nu}(x) \bar{e}^{\rho}(x)$, which follows from the corresponding relation for $\partial_{\mu} e_{a}^{\nu}(x)$. The operator $\bar{Q}$ is then given by Hermitian conjugation, and one can express the result as

$$
\bar{Q}=-\frac{i}{\sqrt{m}} \bar{\psi} e^{\mu}(x)\left[p_{\mu}-A_{\mu}(x)-\frac{1}{2} \omega_{\mu}(x)+\frac{i}{2} \Gamma^{\nu}{ }_{\nu \mu}(x)\right]
$$

The way that the Christoffel symbols and spin connection enter these expressions may appear somewhat perplexing. But it has a simple geometrical origin, which can be seen if one considers the Hilbert space structure of wave functions defined on a curved manifold. This aspect is discussed below.

The differential operators $p_{\mu}=-i \partial_{\mu}$ provide a faithful representation of the Hermitian momentum operators if the inner product of two state vectors $\left|\Psi_{1}\right\rangle$ and $\left|\Psi_{2}\right\rangle$, with corresponding wave functions $\Psi_{1}(x)$ and $\Psi_{2}(x)$, is taken as

$$
\left\langle\Psi_{1} \mid \Psi_{2}\right\rangle=\int d^{2} x \Psi_{1}^{*}(x) \Psi_{2}(x)
$$

Quantum operators obtained by Weyl-ordering the classical expressions may well act on the states the norm of which are defined with the help of this inner product. In a curved surface, however, we have more convenient inner product in the one using the invariant volume measure $\sqrt{g} d^{2} x$, i.e.,

$$
\left\langle\Psi_{1} \mid \Psi_{2}\right\rangle=\int d^{2} x \sqrt{g(x)} \hat{\Psi}_{1}^{*}(x) \hat{\Psi}_{2}(x)
$$

where $\hat{\Psi}(x)$ denotes the 'invariant' wave function associated with the ket $|\Psi\rangle$. Evidently, these differently normalized wavefunctions are related by 


$$
\hat{\Psi}(x)=g^{-1 / 4}(x) \Psi(x)
$$

Then, given a differential operator realization $\Omega$ in the Hilbert space equipped with the inner product (3.5), we are led to consider, if the inner product (3.6) is assumed instead, the differential operator realization

$$
\hat{\Omega}=g^{-1 / 4}(x) \Omega g^{1 / 4}(x)
$$

This becomes nontrivial only for that containing the momentum operator, with $p_{\mu}=-i \partial_{\mu}$ and

$$
\begin{aligned}
\hat{p}_{\mu} & =g^{-1 / 4}(x) p_{\mu} g^{1 / 4}(x)=p_{\mu}-\frac{i}{4} \partial_{\mu}[\log g(x)] \\
& =p_{\mu}-\frac{i}{2} \Gamma_{\nu \mu}^{\nu}(x)
\end{aligned}
$$

For the wave function $\hat{\Psi}(x)$, the differential operators $\hat{p}_{\mu}$ should be used to represent the Hermitian momentum operators. Now, noting (thanks to the anticommutation relations for $\psi, \bar{\psi}))$ that $\pm \frac{1}{2} \omega_{\mu}(x)$ may be replaced by $\frac{1}{2} \omega_{\mu}(x)[\bar{\psi}, \psi]=\omega_{\mu}(x) S(\psi)$, we may write the supercharge operators $\hat{Q}\left(=g^{-1 / 4}(x) Q g^{1 / 4}(x)\right)$ and $\hat{\bar{Q}}\left(=g^{-1 / 4}(x) \bar{Q} g^{1 / 4}(x)\right)$ as

$$
\begin{gathered}
\hat{Q}=\frac{i}{\sqrt{m}} \psi \bar{e}^{\mu}(x)\left[p_{\mu}-A_{\mu}(x)+\omega_{\mu}(x) S(\psi)\right], \\
\hat{\bar{Q}}=-\frac{i}{\sqrt{m}} \bar{\psi} e^{\mu}(x)\left[p_{\mu}-A_{\mu}(x)+\omega_{\mu}(x) S(\psi)\right] .
\end{gathered}
$$

These forms now look quite reasonable. But, in these quantum expressions(cf. the classical ones in $(2.13)$ ), notice the presence of the term coupling the spin connection to the spin operator $S(\psi)$.

From now on we shall assume the inner product given by (3.6) for our wave functions and use the expressions in $(3.10 \mathrm{a}, \mathrm{b})$ for the supercharge operators (with the hats over $Q, \bar{Q}$ now suppressed, but retaining the notation $\left.p_{\mu}=-i \partial_{\mu}\right)$. With the first supercharge operator $Q_{1}=\frac{1}{i}(Q-\bar{Q})$ having the explicit form

$$
Q_{1}=\frac{1}{\sqrt{m}} \psi_{a} e_{a}^{\mu}(x)\left[p_{\mu}-A_{\mu}(x)+\omega_{\mu}(x) S(\psi)\right],
$$

the Hamiltonian operator can then be identified with $H=Q_{1}^{2}$. To write this operator in more familiar form, let us employ the $2 \times 2$ matrix representation for the fermionic operators 
$\psi_{a}=\frac{1}{\sqrt{2}} \sigma_{a}(a=1,2)$, so that we may have $S(\psi)=\frac{1}{2} \sigma_{3}$. Then the supercharge $Q_{1}$, acting on a two-component spinor wave function, acquires the form

$$
Q_{1}=\frac{1}{\sqrt{2 m}} \sigma_{a} e_{a}^{\mu}(x)\left[-i \partial_{\mu}-A_{\mu}+\frac{1}{2} \sigma_{3} \omega_{\mu}(x)\right] \equiv \frac{1}{\sqrt{2 m}} \sigma_{a} e_{a}^{\mu}(x)\left(-i D_{\mu}\right),
$$

where $D_{\mu}$ is the covariant derivative which include both the gauge- and spin- connection terms. We now find the Hamiltonian operator

$$
\begin{aligned}
H & =-\frac{1}{2 m}\left[\sigma_{a} e_{a}^{\mu}(x) D_{\mu}\right]^{2} \\
& =-\frac{1}{2 m} \frac{1}{\sqrt{g}} D_{\mu} \sqrt{g} g^{\mu \nu} D_{\nu}-\frac{1}{2 m} \sigma_{3} B(x)+\frac{1}{8 m} R(x),
\end{aligned}
$$

where $R(x) \equiv \epsilon_{a b} e_{a}^{\mu}(x) e_{a}^{\nu}(x) R_{\mu \nu}(x)$, with $R_{\mu \nu}(x)=\partial_{\mu} \omega_{\nu}(x)-\partial_{\nu} \omega_{\mu}(x)$, is the Ricci scalar for the surface. [Note that, in two dimensions, the Riemann curvature contains only the pieces linear in $\left.\omega_{\mu a b}\right]$. As one can easily verify, the operators $H, Q$ and $\bar{Q}$ we have just constructed satisfy the $N=2$ supersymmetry algebra:

$$
\begin{aligned}
& \{Q, Q\}=\{\bar{Q}, \bar{Q}\}=0, \\
& \{Q, \bar{Q}\}=H \\
& {[H, Q]=[H, \bar{Q}]=0 .}
\end{aligned}
$$

We also remark that, with $\psi_{a}=\frac{1}{\sqrt{2}} \sigma_{a}$, matrices for $\psi$ and $\bar{\psi} \operatorname{read} \psi=\left(\begin{array}{cc}0 & 0 \\ 1 & 0\end{array}\right) \equiv \sigma_{-}$and $\bar{\psi}=\left(\begin{array}{ll}0 & 1 \\ 0 & 0\end{array}\right) \equiv \sigma_{+}$

The matrix Hamiltonian (3.13), the curved-space generalization of the flat-space Pauli Hamiltonian in (1.1), should be relevant apparently for a spin-1/2 particle. But, in nonrelativistic quantum mechanics, 'spin' is nothing more than another internal degrees of freedom; in this light, the above Hamiltonian may well be reinterpreted just as that containing a pair of Schrödinger Hamiltonains considered for a scalar particle. For the purpose, we employ the above $2 \times 2$ matrix representations for $\psi, \bar{\psi}$ with the supercharge operators in $(3.10 \mathrm{a}, \mathrm{b})$, to write 


$$
\begin{aligned}
H & =Q \bar{Q}+\bar{Q} Q \\
& =\frac{1}{m}\left(\begin{array}{cc}
\pi_{-} \pi_{+} & 0 \\
0 & \pi_{+} \pi_{-}
\end{array}\right) \equiv\left(\begin{array}{cc}
H_{+} & 0 \\
0 & H_{-}
\end{array}\right),
\end{aligned}
$$

where we have defined

$$
\pi_{+} \equiv \bar{e}^{\mu}(x)\left[p_{\mu}-A_{\mu}(x)+\frac{1}{2} \omega_{\mu}(x)\right], \pi_{-} \equiv e^{\mu}(x)\left[p_{\mu}-A_{\mu}(x)-\frac{1}{2} \omega_{\mu}(x)\right]
$$

In (3.15) we have two Hamiltonians $H_{ \pm}=\frac{1}{m} \pi_{\mp} \pi_{ \pm}$connected by supersymmetry transformations. As we set $\mathcal{A}_{\mu}^{+}=A_{\mu}-\frac{1}{2} \omega_{\mu}$, it is also evident from (3.13) that the Hamiltonian $H_{+}$can be expressed by the form

$$
H_{+}=-\frac{1}{2 m} \frac{1}{\sqrt{g}} D_{\mu}^{+} \sqrt{g} g^{\mu \nu} D_{\nu}^{+}-\frac{1}{2 m} \mathcal{B}^{+}(x),
$$

where $D_{\mu}^{+}=\partial_{\mu}-i \mathcal{A}_{\mu}^{+}(x)$, and $\mathcal{B}^{+}$is the magnetic field obtained from the vector potential $\mathcal{A}_{\mu}^{+}$. Similarly, writing $\mathcal{A}_{\mu}^{-} \equiv A_{\mu}+\frac{1}{2} \omega_{\mu}$, we can express the second Hamiltonian $H_{-}$as

$$
H_{-}=-\frac{1}{2 m} \frac{1}{\sqrt{g}} D_{\mu}^{-} \sqrt{g} g^{\mu \nu} D_{\nu}^{-}+\frac{1}{2 m} \mathcal{B}^{-}(x),
$$

with $D_{\mu}^{-}=\partial_{\mu}-i \mathcal{A}_{\mu}^{-}(x)$. From this discussion it follows that our system contains a supersymmetric pair of curved-space scalar Hamiltonians, i.e., $H_{+}$and $H_{-}$, with $H_{+}\left(H_{-}\right)$ defined in the simultaneous presence of the vector potential $\mathcal{A}_{\mu}^{+}\left(\mathcal{A}_{\mu}^{-}\right)$and the scalar po-

tential $-\frac{1}{2 m} \mathcal{B}^{+}\left(+\frac{1}{2 m} \mathcal{B}^{-}\right)$. The problem based on this view and that based on the spin- $1 / 2$ particle interpretation are mathematically equivalent; one can go from one problem to the other by identifying things like potentials differently. It also follows, from the supersymmetry algebra, that the above Hamiltonian $H_{ \pm}$admit only non-negative eigenvalues.

\section{COMPLETE ENERGY EIGENSTATES FOR SHAPE-INVARIANT SYSTEMS}

The supersymmetric pair Hamiltonian structure found in the previous section can be used to find the full Hilbert space (i.e., complete energy eigenstates) for the Landau system defined on the two-sphere or on the hyperbolic plane. This becomes possible because, in the 
case of a constant magnetic field present over a constant-curvature surface, the corresponding Hamiltonian acquires additionally the so-called shape-invariance porperty $[11,12]$ which allows one to develop simple algebraic methods to find the spectrum and energy eigenstates. [But, in contrast to the one-dimensional examples considered in Refs. [11,12], we here have two-dimensional shape-invariant systems]. In what follows, we will assume that the Hamiltonian we wish to really study is $H_{+}$(as given by (3.17)), with $\mathcal{A}_{\mu}^{+}(x)$ now renamed as $A_{\mu}(x)$ (and, correspondingly, $\mathcal{A}_{\mu}^{-}(x)$ as $A_{\mu}(x)+\omega_{\mu}(x)$ ). Also, in the present situation where $B(x)$ and $R(x)$ are constant, $F_{\mu \nu}(x)\left(=\partial_{\mu} A_{\nu}(x)-\partial_{\nu} A_{\mu}(x)\right)$ and $R_{\mu \nu}(x)\left(=\partial_{\mu} \omega_{\nu}(x)-\partial_{\nu} \omega_{\mu}(x)\right)$ are both proportional to $\epsilon_{\mu \nu}\left(=e_{\mu}^{a} e_{\nu}^{b} \epsilon_{a b}\right)$ and so we may write, with a judicious choice of the zweibeins,

$$
A_{\mu}(x)=\gamma \omega_{\mu}(x)
$$

where $\gamma$ is a constant. We remark that $\gamma$ can be any real number in the case of the hyperbolic plane. But, in the two-sphere case, the famous Dirac quantization condition[22] for a globally well-defined one-form potential demands that $\gamma$ be restricted to half-integral values.

Let us denote the vector potential in (4.1) by $A_{\mu}^{\gamma}(x)$, and the corresponding magnetic field strength by $B^{\gamma}\left(=\frac{1}{2} \gamma R\right.$, if $R$ is the constant scalar curvature of the surface). We write the Hamiltonian $H_{+}\left(\right.$in $(\underline{3.17})$ ) with $A_{\mu}^{\gamma}(x)$ taking the place of $\mathcal{A}_{\mu}^{+}(x)$ as $\mathcal{H}_{\gamma}$, i.e.,

$$
\mathcal{H}_{\gamma}=-\frac{1}{2 m} \frac{1}{\sqrt{g(x)}} D_{\mu}^{\gamma} \sqrt{g(x)} g^{\mu \nu}(x) D_{\nu}^{\gamma}-\frac{1}{2 m} B^{\gamma}, \quad\left(D_{\mu}^{\gamma} \equiv \partial_{\mu}-i A_{\mu}^{\gamma}\right) .
$$

But for the trivial additive constant $\frac{1}{2 m} B^{\gamma}, \mathcal{H}_{\gamma}$ is really the Landau Hamiltonian on the surface. We know that $\mathcal{H}_{\gamma}$ may also be written in the form

$$
\mathcal{H}_{\gamma}=\frac{1}{m} \pi_{\gamma+1} \bar{\pi}_{\gamma}
$$

where we defined the operators $\bar{\pi}_{\gamma}, \pi_{\gamma+1}$ according to (see (3.16))

$$
\pi_{\gamma} \equiv e^{\mu}(x)\left[p_{\mu}-A_{\mu}^{\gamma}(x)\right], \quad \bar{\pi}_{\gamma} \equiv \bar{e}^{\mu}(x)\left[p_{\mu}-A_{\mu}^{\gamma}(x)\right]
$$

and used the fact that $A_{\mu}^{\gamma+1}(x)=A_{\mu}^{\gamma}(x)+\omega_{\mu}(x)$. On the other hand, from (3.18) and the relation $H_{-}=\frac{1}{m} \pi_{+} \pi_{-}$, the superpartner Hamiltonian related to $\mathcal{H}_{\gamma}$ equals 


$$
\begin{aligned}
\frac{1}{m} \bar{\pi}_{\gamma} \pi_{\gamma+1} & =-\frac{1}{2 m} \frac{1}{\sqrt{g(x)}} D_{\mu}^{\gamma+1} \sqrt{g(x)} g^{\mu \nu}(x) D_{\nu}^{\gamma+1}+\frac{1}{2 m} B^{\gamma+1} \\
& =\mathcal{H}_{\gamma+1}+\frac{1}{m} B^{\gamma+1},
\end{aligned}
$$

(or $\frac{1}{m} \bar{\pi}_{\gamma-1} \pi_{\gamma}=\mathcal{H}_{\gamma}+\frac{1}{m} B^{\gamma}$ ). Now suppose that $|E\rangle^{\gamma}$ is an eigenstate of $\mathcal{H}_{\gamma}$ with eigenvalue $E$. Then, from (4.3) and (4.5), we are led to the relation

$$
\begin{aligned}
\mathcal{H}_{\gamma+1} \bar{\pi}_{\gamma}|E\rangle^{\gamma} & =\left(\frac{1}{m} \bar{\pi}_{\gamma} \pi_{\gamma+1}-\frac{1}{m} B^{\gamma+1}\right) \bar{\pi}_{\gamma}|E\rangle^{\gamma}=\bar{\pi}_{\gamma}\left(\mathcal{H}_{\gamma}-\frac{1}{m} B^{\gamma+1}\right)|E\rangle^{\gamma} \\
& =\left(E-\frac{1}{m} B^{\gamma+1}\right) \bar{\pi}_{\gamma}|E\rangle^{\gamma}
\end{aligned}
$$

i.e., $\bar{\pi}_{\gamma}|E\rangle^{\gamma}$ is an eigenstate of $\mathcal{H}_{\gamma+1}$ with energy $E-\frac{1}{m} B^{\gamma+1}$. Analogously, for $\pi_{\gamma}|E\rangle^{\gamma}$, we find

$$
\mathcal{H}_{\gamma-1} \pi_{\gamma}|E\rangle^{\gamma}=\frac{1}{m} \pi_{\gamma} \bar{\pi}_{\gamma-1} \pi_{\gamma}|E\rangle^{\gamma}=\left(E+\frac{1}{m} B^{\gamma}\right) \pi_{\gamma}|E\rangle^{\gamma}
$$

i.e., $\pi_{\gamma}|E\rangle^{\gamma}$ is an eigenstate of $\mathcal{H}_{\gamma-1}$ with eigenvalue $E+\frac{1}{m} B^{\gamma}$.

The particular structure shown in (4.3) and (4.5) describes the shape-invariance property of our Hamiltonian $\mathcal{H}_{\gamma}$ (with parameter $\gamma$ ), which we will utilize to find the complete energy eigenstates. Without loss of generality we may here assume $B^{\gamma}=\frac{1}{2} \gamma R>0$; this corresponds to the choice $\gamma>0$ if $R>0$ (i.e., in the case of the two-sphere), and $\gamma<0$ if $R<0$ (the hyperbolic plane). Now, for any given eigenstate $|E\rangle^{\gamma}$ of $\mathcal{H}_{\gamma}$, we know from this shapeinvariance property that $\bar{\pi}_{\gamma+n-1} \bar{\pi}_{\gamma+n-2} \cdots \bar{\pi}_{\gamma}|E\rangle^{\gamma}$, if it is not a zero vector, should correspond to an eigenstate of $\mathcal{H}_{\gamma+n}$ with energy equal to $E-\frac{1}{m} B^{\gamma+1} \cdots-\frac{1}{m} B^{\gamma+n}$. In the case of the two-sphere where $B^{\gamma+n}=\frac{1}{2}(\gamma+n) R>0$ for all $n=0,1,2, \cdots$, this procedure of generating new states must stop after applying a finite number of $\bar{\pi}$ 's (since the eigenvalue of $\mathcal{H}_{\gamma+n}$ for any $n \geq 0$ cannot be negative); i.e., for any $|E\rangle^{\gamma}$, there exists some non-negative integer $s$ such that

$$
\bar{\pi}_{\gamma+s} \bar{\pi}_{\gamma+s-1} \cdots \bar{\pi}_{\gamma}|E\rangle^{\gamma}=0
$$

while $\bar{\pi}_{\gamma+s-1} \bar{\pi}_{\gamma+s-2} \cdots \bar{\pi}_{\gamma}|E\rangle^{\gamma} \neq 0$. When (4.8) holds, $\bar{\pi}_{\gamma+s-1} \bar{\pi}_{\gamma+s-2} \cdots \bar{\pi}_{\gamma}|E\rangle^{\gamma}$ corresponds to a zero-energy eigenstate of $\mathcal{H}_{\gamma+s}=\pi_{\gamma+s+1} \bar{\pi}_{\gamma+s}\left(\right.$ i.e., $\bar{\pi}_{\gamma+s-1} \bar{\pi}_{\gamma+s-2} \cdots \bar{\pi}_{\gamma}|E\rangle^{\gamma} \propto|0\rangle^{\gamma+s}$ ) and therefore 


$$
E-\frac{1}{m} B^{\gamma+1}-\cdots-\frac{1}{m} B^{\gamma+s}=0
$$

The relation (4.9) determines the eigenvalue $E$ of $\mathcal{H}_{\gamma}$ in terms of the quantum number $s$, showing also that we have a discrete spectrum only. In the case of the hyperbolic plane (with $R<0$ and $\gamma<0$ ), however, the situation is somewhat different since $B^{\gamma+n}=\frac{1}{2} \gamma R+\frac{1}{2} n R$ changes sign from the positive to the negative for large enough $n$. In the latter case there exist not only discrete energy levels (determined by the condition like (4.9)) but also a continuous spectrum for energy exceeding some critical value[13]. We shall below construct the complete energy eigenstates for the two cases separately.

\section{A.Complete Energy Eigenstates on the Two-sphere}

Here, as we remarked already, $\gamma(>0)$ must be half integral. Since there exists a discrete spectrum only, we may denote the eigenstates of $\mathcal{H}_{\gamma}$ by $\left|E_{s}\right\rangle^{\gamma}$. Allowed values of $E_{s}$ - the eigenvalue spectrum of $\mathcal{H}_{\gamma}$ - are determined by solving (4.9):

$$
E_{s}=\frac{1}{m} \sum_{k=1}^{s} B^{\gamma+k}=\frac{R}{4 m} s(2 \gamma+s+1), \quad(s=0,1,2, \cdots) .
$$

To find the full Hilbert space, we may begin with determining the general wave functions corresponding to the states $|0\rangle^{\gamma+s}$. This is a relatively easy task, for they have the property of being annihilated by the first-order operator, that is,

$$
\bar{\pi}_{\gamma+s}|0\rangle^{\gamma+s}=0
$$

Once they are known, we may use (4.7): this relation tells us that $\left|E_{s}\right\rangle^{\gamma}$ may be obtained simply by acting $\pi_{\gamma+1} \pi_{\gamma+2} \cdots \pi_{\gamma+s}$ on $|0\rangle^{\gamma+s}$. Indeed, assuming that $|0\rangle^{\gamma+s}$ is normalized, we can obtain the correctly normalized states $\left|E_{s}\right\rangle^{\gamma}$ through

$$
\left|E_{s}\right\rangle^{\gamma}=\sqrt{\frac{\Gamma(2 \gamma+s+1)}{\Gamma(s+1) \Gamma(2 \gamma+2 s+1)}} \pi_{\gamma+1} \pi_{\gamma+2} \cdots \pi_{\gamma+s}|0\rangle^{\gamma+s} .
$$

(One may recall our earlier finding $|0\rangle^{\gamma+s} \propto \bar{\pi}_{\gamma+s-1} \bar{\pi}_{\gamma+s-2} \cdots \bar{\pi}_{\gamma}\left|E_{s}\right\rangle^{\gamma}$ ). To fix the normalization constant in (4.12), we have made use of the relations in (4.3)-(4.7). 
Because of the shape-invariance in the system, one can thus reduce the problem of finding complete energy eigenfunctions for a given magnetic field strength essentially to that of finding zero-energy eigenfunctions for different magnetic field strengths or to the analysis of (4.11). For explicit eigenfunctions, suitable coordinates must be chosen[Remember that our wave function defines a scalar field under coordinate transformation]. To obtain explicit zero-energy wave functions using spherical coordinates $\theta, \phi$ for instance, we may consider the one-forms $\mathbf{e}^{a}$ given by

$$
\begin{aligned}
& \mathbf{e}^{a}=\Lambda(\Theta)_{b}^{a} \mathbf{e}_{(0)}^{b}, \\
& \Lambda(\Theta)=\left(\begin{array}{cc}
\cos \Theta(\theta, \phi), & -\sin \Theta(\theta, \phi) \\
\sin \Theta(\theta, \phi), & \cos \Theta(\theta, \phi)
\end{array}\right) \in S O(2)
\end{aligned}
$$

where $\mathbf{e}_{(0)}^{a}$ are the usually chosen one-forms related to the line element $g_{\mu \nu} d x^{\mu} d x^{\nu}=r^{2} d \theta^{2}+$ $r^{2} \sin ^{2} \theta d \phi^{2}$ (the constant $r$ here represents the radius of the sphere), i.e.,

$$
\begin{aligned}
& \mathbf{e}_{(0)}^{1} \equiv e_{(0) \theta}^{1} d \theta+e_{(0) \phi}^{1} d \phi=r d \theta, \\
& \mathbf{e}_{(0)}^{2} \equiv e_{(0) \theta}^{2} d \theta+e_{(0) \phi}^{2} d \phi=r \sin \theta d \phi .
\end{aligned}
$$

The local frame rotation $\Lambda(\Theta)$ above are to be chosen such that the vector potentials $A_{\mu}$ as given by (4.1) may acquire most convenient forms. Calculating the spin connections using the form (4.13) yields

$$
\omega_{\mu}=\omega_{(0) \mu}+\partial_{\mu} \Theta
$$

with $\omega_{(0) \theta}=0$ and $\omega_{(0) \phi}=-\cos \theta$, and for the scalar curvature we find the value $R=\frac{2}{r^{2}}$. We now choose $\Theta(\theta, \phi)=\phi$. Then

$$
\omega_{\theta}=0, \omega_{\phi}=1-\cos \theta
$$

and from (4.1) we obtain the familiar magnetic-monopole vector potentials[22]

$$
A_{\theta}=0, \quad A_{\phi}=\gamma(1-\cos \theta),
$$

corresponding to the constant magnetic field strength $B=\frac{\gamma}{r^{2}}$. 
Based on the above information, one has the operator $\bar{\pi}_{\gamma}$ in spherical coordinates expressed by the form

$$
\bar{\pi}_{\gamma}=-\frac{i}{\sqrt{2} r} e^{i \phi}\left\{\left(\partial_{\theta}+\gamma \frac{1-\cos \theta}{\sin \theta}\right)+\frac{i}{\sin \theta} \partial_{\phi}\right\} .
$$

Evidently, acting on an eigenstate of the angular momentum $-i \partial_{\phi}$ (with eigenvalue $l=$ $0, \pm 1, \pm 2, \cdots)$, the operator $\bar{\pi}_{\gamma}$ will have the effect of raising the value of $l$ by 1 . Let

$$
\Psi_{s, l}^{\gamma}(\theta, \phi) \equiv\left\langle\theta, \phi \mid E_{s}, l\right\rangle^{\gamma}=\Phi_{s, l}^{\gamma}(\theta) e^{i l \phi}
$$

denote the normalized eigenfunction of $\mathcal{H}_{\gamma}$ with energy $E_{s}($ in $(4.10))$ and angular momentum l. Then, from (4.19),

$$
\bar{\pi}_{\gamma} \Psi_{s, l}^{\gamma}(\theta, \phi)=\left[\bar{\pi}_{\gamma}^{(l)} \Psi_{s, l}^{\gamma}(\theta)\right] e^{i(l+1) \phi},
$$

where the operator $\bar{\pi}_{\gamma}^{(l)}$ is given as

$$
\begin{aligned}
\bar{\pi}_{\gamma}^{(l)} & =-\frac{i}{\sqrt{2} r}\left(\partial_{\theta}+\gamma \frac{1-\cos \theta}{\sin \theta}-\frac{l}{\sin \theta}\right) \\
& =\frac{i}{\sqrt{2} r}(1-\cos \theta)^{\frac{l+1}{2}}(1+\cos \theta)^{\gamma+1-\frac{l+1}{2}} \frac{\vec{\partial}}{\partial(\cos \theta)}(1-\cos \theta)^{-\frac{l}{2}}(1+\cos \theta)^{-\gamma+\frac{l}{2}}
\end{aligned}
$$

Now, if $\Psi_{(0), l}^{\gamma+s}(\theta, \phi)=\Phi_{(0), l}^{\gamma+s}(\theta) e^{i l \phi}$ corresponds to a zero-energy state of $\mathcal{H}_{\gamma+\rho}$, we know from $(4.11)$ and (4.21) that $\Phi_{(0), l}^{\gamma+s}(\theta)$ must satisfy the equation $\bar{\pi}_{\gamma+s}^{(l)} \Phi_{(0), l}^{\gamma+s}(\theta)=0$. Then, using (4.22) for $\bar{\pi}_{\gamma+s}^{(l)}$, it follows that $\Phi_{(0), l}^{\gamma+s}(\theta)$ is proportional to $(1-\cos \theta)^{\frac{l}{2}}(1+\cos \theta)^{\gamma+s-\frac{l}{2}}$. From this discussion the desired normalized wave function $\Psi_{(0), l}^{\gamma+s}(\theta, \phi)$ is found to have the explicit form

$$
\begin{array}{r}
\Psi_{(0), l}^{\gamma+s}(\theta, \phi)=\frac{1}{2^{\gamma+s}} \sqrt{\frac{\Gamma(2 \gamma+2 s+2)}{4 \pi \Gamma(2 \gamma+2 s-l+1) \Gamma(l+1)}}(1-\cos \theta)^{\frac{l}{2}}(1+\cos \theta)^{\gamma+s-\frac{l}{2}} e^{i l \phi}, \\
(l=0,1, \cdots, 2 \gamma+2 s)
\end{array}
$$

where the restriction on the quantum number $l$ derives from the normalizability requirement. All that remains is to use (4.12) to find the complete normalized eigenfunctions of $\mathcal{H}_{\gamma}$. Here, since the operator $\pi_{\gamma}$ has the effect of lowering the angular momentum value by 1 , one can facilitate the calculation by introducing the operator analogous to $\bar{\pi}_{\gamma}^{(l)}$ (in (4.22)); it equals the expression 


$$
\pi_{\gamma}^{(l)}=\frac{i}{\sqrt{2} r}(1-\cos \theta)^{-\frac{l-1}{2}}(1+\cos \theta)^{-(\gamma-1)+\frac{l-1}{2}} \frac{\vec{\partial}}{\partial(\cos \theta)}(1-\cos \theta)^{\frac{l}{2}}(1+\cos \theta)^{\gamma-\frac{l}{2}} .
$$

The effect of applying $\pi_{\gamma+1} \cdots \pi_{\gamma+s-1} \pi_{\gamma+s}$ on $\Psi_{(0), l}^{\gamma+s}(\theta, \phi)=\Phi_{(0), l}^{\gamma+s}(\theta) e^{i l \phi}$ (see 4.12)) can then be described by the action of $\pi_{\gamma+1}^{(l-s)} \cdots \pi_{\gamma+s-1}^{(l-1)} \pi_{\gamma+s}^{(l)}$ on $\Phi_{(0), l}^{\gamma+s}(\theta)$ combined with the change $e^{i l \phi} \rightarrow e^{i(l-s) \phi}$. The result is the following form for complete energy eigenfunctions(which form 'monopole harmonics'):

$$
\begin{array}{r}
\Psi_{s, l}^{\gamma}(\theta, \phi)=\frac{1}{2^{\gamma}} \sqrt{\frac{(2 \gamma+2 s+1) \Gamma(s+1) \Gamma(2 \gamma+s+1)}{4 \pi \Gamma(l+s+1) \Gamma(2 \gamma-l+s+1)}}(1-\cos \theta)^{\frac{l}{2}}(1+\cos \theta)^{\gamma-\frac{l}{2}} P_{s}^{(l, 2 \gamma-l)}(\cos \theta) e^{i l \phi}, \\
(s=0,1, \cdots ; l=-s,-s+1, \cdots, 2 \gamma+s),
\end{array}
$$

where

$$
P_{s}^{(\alpha, \beta)}(x)=\frac{1}{2^{s} \Gamma(s+1)}(1-x)^{-\alpha}(1+x)^{-\beta}\left(\frac{d}{d x}\right)^{s}(1-x)^{\alpha+s}(1+x)^{\beta+s}
$$

is the Jacobi polynomial[23].

If one wishes, the above analysis may be carried out using the complex coordinates $z, \bar{z}$ which are related to the spherical coordinates by the stereographic projection

$$
z=\left(\tan \frac{\theta}{2}\right) e^{i \phi}, \bar{z}=\left(\tan \frac{\theta}{2}\right) e^{-i \phi}
$$

Then the operators $\pi_{\gamma}, \bar{\pi}_{\gamma}$ become

$$
\begin{aligned}
\pi_{\gamma} & =-\frac{i}{\sqrt{2} r}\left\{(1+z \bar{z}) \partial_{z}-\gamma \bar{z}\right\}=-\frac{i}{\sqrt{2} r}(1+z \bar{z})^{\gamma+1} \vec{\partial}_{z}(1+z \bar{z})^{-\gamma} \\
\bar{\pi}_{\gamma}=-\frac{i}{\sqrt{2} r}\left\{(1+z \bar{z}) \partial_{\bar{z}}+\gamma z\right\} & =-\frac{i}{\sqrt{2} r}(1+z \bar{z})^{-\gamma+1} \vec{\partial}_{\bar{z}}(1+z \bar{z})^{\gamma}
\end{aligned}
$$

Since the complete energy eigenfunctions can be found in much the same way as above (see also Ref.[14]), we shall here give the final expression only. It reads

$$
\Psi_{s, l}^{\gamma}(z, \bar{z})=\sqrt{\frac{(2 \gamma+2 s+1) \Gamma(2 \gamma+s+1)}{4 \pi \Gamma(s+1) \Gamma(l+s+1) \Gamma(2 \gamma-l+s+1)}}(1+z \bar{z})^{\gamma}\left[(1+z \bar{z})^{2} \frac{\partial}{\partial z}\right]^{s} \frac{z^{l+s}}{(1+z \bar{z})^{2(\gamma+s)}},
$$

or, in terms of the Jacobi function, we have (note that $\cos \theta=\frac{1-z \bar{z}}{1+z \bar{z}}$ )

$$
\Psi_{s, l}^{\gamma}(z, \bar{z})=\sqrt{\frac{(2 \gamma+2 s+1) \Gamma(s+1) \Gamma(2 \gamma+s+1)}{4 \pi \Gamma(l+s+1) \Gamma(2 \gamma-l+s+1)}} \frac{z^{l}}{(1+z \bar{z})^{\gamma}} P_{s}^{(l, 2 \gamma-l)}\left(\frac{1-z \bar{z}}{1+z \bar{z}}\right) .
$$

This is nothing but the wavefunction (4.25) written in terms of the coordinates $z$ and $\bar{z}$. 


\section{B.Complete Energy Eigenstates on the Hyperbolic Plane}

When the scalar curvature $R$ is a negative constant, the eigenstates $|E\rangle^{\gamma}$ of $\mathcal{H}_{\gamma}$ (with $\gamma$ taken to be negative) come in two distinct families. The first, a discrete family, corresponds to those with energy $E$ not exceeding the appropriate critical value so that there exists some non-negative integer $s$ realizing the annihilation condition (4.8), and the second to those with energy eigenvalue exceeding the critical value. One can readily find the critical value from studying (4.9). Here, using $B^{\gamma+k}=-\frac{1}{2}|R|(-|\gamma|+k)$, we have

$$
\begin{aligned}
\frac{1}{m} \sum_{k=1}^{s} B^{\gamma+k} & =\frac{|R|}{4 m} s(2|\gamma|-s-1) \\
& =-\frac{|R|}{4 m}\left[s-\left(|\gamma|-\frac{1}{2}\right)\right]^{2}+\frac{|R|}{4 m}\left(|\gamma|-\frac{1}{2}\right)^{2},
\end{aligned}
$$

and hence the right hand side of (4.9) remains positive for any $s$-value if $E>\frac{|R|}{4 m}\left(|\gamma|-\frac{1}{2}\right)^{2}$, i.e., no solution to (4.9) exists. On the other hand, for $E$ smaller than the critical value $\frac{|R|}{4 m}\left(|\gamma|-\frac{1}{2}\right)^{2}$, the condition (4.9) serves to determine the allowed energy eigenvalues just as in the case of the two-sphere; this yields the discrete spectrum

$$
E_{s}=\frac{|R|}{4 m} s(2|\gamma|-s-1),\left(s=0,1,2, \cdots,\left[|\gamma|-\frac{1}{2}\right]\right) .
$$

If $E>\frac{|R|}{4 m}\left(|\gamma|-\frac{1}{2}\right)^{2}$, no such restriction exists and we expect a continuous spectrum[13]. We shall below find the complete energy eigenfunctions which are associated with these two distinct families.

For the energy eigenfunctions corresponding to the discrete spectrum (4.33), we may first find the zero-energy eigenfunctions of $\mathcal{H}_{-|\gamma|+s}$ (i.e., those corresponding to the states $|0\rangle^{-|\gamma|+s}$ in the specific coordinates chosen), and then use the formula analogous to (4.12) to obtain the eigenfunctions corresponding to the states $\left|E_{s}\right\rangle^{\gamma}$. Let us choose the hyperbolic coordinates $\theta$, $\phi$ (with $0 \leq \theta<\infty, 0 \leq \phi<2 \pi$ ) in terms of which the line element is described by the Lobachevsky metric $d s^{2}=r^{2} d \theta^{2}+r^{2} \sinh ^{2} \theta d \phi^{2}$ (for a real constant r) [24] The one-forms $\mathbf{e}^{a}$ may still be given by (4.13), if $\mathbf{e}_{(0)}^{a}$ in the present case are taken to be (cf.(4.14))

$$
\mathbf{e}_{(0)}^{1}=r d \theta \quad, \quad \mathbf{e}_{(0)}^{2}=r \sinh \theta d \phi .
$$


We here have the scalar curvature $R=-\frac{2}{r^{2}}$, and the vector potentials $A_{\mu}\left(=-|\gamma| \omega_{\mu}\right)$ assume the form

$$
A_{\theta}=0 \quad, \quad A_{\phi}=|\gamma|(\cosh \theta-1)
$$

The operators $\bar{\pi}_{\gamma}, \pi_{\gamma}$ in hyperbolic coordinates are then found readily. We may also represent the normalized eigenfunction of $\mathcal{H}_{\gamma}$ with energy $E_{s}$ and angular momentum $l$ by $\Psi_{s, l}^{\gamma}(\theta, \phi)=$ $\Phi_{s, l}^{\gamma}(\theta) e^{i l \phi}$, and go on to introduce the operators $\bar{\pi}_{\gamma}^{(l)}, \pi_{\gamma}^{(l)}$ as in the previous two-sphere case:

$$
\begin{aligned}
& \bar{\pi}_{\gamma}^{(l)}=-\frac{i}{\sqrt{2} r}(\cosh \theta-1)^{\frac{l+1}{2}}(\cosh \theta+1)^{-|\gamma|+1-\frac{l+1}{2}} \frac{\vec{\partial}}{\partial(\cosh \theta)}(\cosh \theta-1)^{-\frac{l}{2}}(\cosh \theta+1)^{|\gamma|+\frac{l}{2}} \\
& \pi_{\gamma}^{(l)}=-\frac{i}{\sqrt{2} r}(\cosh \theta-1)^{-\frac{l-1}{2}}(\cosh \theta+1)^{|\gamma|+1+\frac{l-1}{2}} \frac{\vec{\partial}}{\partial(\cosh \theta)}(\cosh \theta-1)^{\frac{l}{2}}(\cosh \theta+1)^{-|\gamma|-\frac{l}{2}}
\end{aligned}
$$

Then the eigenfunctions $\Psi_{s, l}^{\gamma}(\theta, \phi)$ are constructed by the methods parallel to those used in the two-sphere case. The results read

$$
\begin{array}{r}
\Psi_{s, l}^{\gamma}(\theta, \phi)=\frac{1}{2^{-|\gamma|}} \sqrt{\frac{(2|\gamma|-2 s-1) \Gamma(s+1) \Gamma(2|\gamma|+l-s)}{4 \pi \Gamma(l+s+1) \Gamma(2|\gamma|-s)}}(\cosh \theta-1)^{\frac{l}{2}}(\cosh \theta+1)^{-|\gamma|-\frac{l}{2}} \tilde{P}_{s}^{(l,-2|\gamma|-l)(\cosh \theta) e^{i l \phi},} \\
\left(s=0,1, \cdots,\left[|\gamma|-\frac{1}{2}\right] ; l=-s,-s+1,-s+2, \cdots\right),
\end{array}
$$

where $\tilde{P}_{s}^{(\alpha, \beta)}(x)$ is the associated Jacobi polynomial[23] given by

$$
\tilde{P}_{s}^{(\alpha, \beta)}(x)=\frac{1}{2^{s} \Gamma(s+1)}(x-1)^{-\alpha}(x+1)^{-\beta}\left(\frac{d}{d x}\right)^{s}(x-1)^{\alpha+s}(x+1)^{\beta+s} .
$$

Note that, in this (noncompact) hyperbolic-plane case, there are an infinite number of allowed $l$ values for given $s$ and this of course implies that each discrete energy level comes with infinite degeneracy here.

To obtain continuum energy eigenfunctions with $E>\frac{|R|}{4 m}\left(|\gamma|-\frac{1}{2}\right)^{2}$, one can resort to the analytic continuation procedure with the above expression for discrete states, following the strategy of Ref.[25] for general shape-invariant systems. Here it is useful to note that our eigenfunction in (4.38) can be rewritten in terms of the hypergeometric function $F(a, b ; c ; x)$, based on the known connection[23] between the latter and the (associated) Jacobi function: 


$$
\begin{aligned}
\Psi_{s, l}^{\gamma}(\theta, \phi) \propto & (\cosh \theta-1)^{\frac{l}{2}}(\cosh \theta+1)^{-|\gamma|-\frac{l}{2}} \\
& \times \frac{\Gamma(s+l+1)}{\Gamma(s+1) \Gamma(l+1)} F\left(-s,-2|\gamma|+s+1 ; l+1 ; \frac{1-\cosh \theta}{2}\right) e^{i l \phi} .
\end{aligned}
$$

[In this form it should be understood that $\frac{1}{\Gamma(l+1)} F\left(-s,-2|\gamma|+s+1 ; l+1 ; \frac{1-\cosh \theta}{2}\right)$ for $l=-s,-s+1, \cdots,-1$ are given by the appropriate limiting expressions as $l$ approaches negative integer values]. The expression (4.40) has the following implication: if one writes

$$
\Psi_{s, l}^{\gamma}(\theta, \phi)=(\cosh \theta-1)^{\frac{l}{2}}(\cosh \theta+1)^{-|\gamma|-\frac{l}{2}} F_{s, l}^{\gamma}(\theta) e^{i l \phi},
$$

the Schrödinger equation for $\Psi_{s, l}^{\gamma}(\theta, \phi)$ reduces to a hypergeometric equation for the function $F_{s, l}^{\gamma}(\theta)$. Now, in this hypergeometric equation for $F_{s, l}^{\gamma}(\theta)$, one might wish to dispense with $s$ in favor of the energy $E(s)=\frac{|R|}{4} s(2|\gamma|-s-1)$; the resulting differential equation would be just the condition on $F_{s, l}^{\gamma}(\theta, \phi)$ (with $s$ related to $\mathrm{E}$ as we prescribed), for the function $\Psi_{s, l}^{\gamma}(\theta, \phi)$ given by (4.41) to describe an energy eigenstate with energy $E$. This equation should be valid for general energy eigenvalue $E$, and it is in this context that we can study the continuum states also within our approach.

For real $E$ but larger than the critical value $\frac{|R|}{4 m}\left(|\gamma|-\frac{1}{2}\right)^{2}$, we have two complex roots $s_{ \pm}$ to the equation $\frac{|R|}{4 m} s(2|\gamma|-s-1)=E$, viz.,

$$
s_{ \pm}(E)=|\gamma|-\frac{1}{2} \pm i \sqrt{\frac{4 m E}{|R|}-\left(|\gamma|-\frac{1}{2}\right)^{2}} .
$$

Then, based on the above discussion, we may simply take the discrete energy eigenfunction $\Psi_{s, l}^{\gamma}(\theta, \phi)$ in (4.40) but with $s$ replaced by the complex value $s_{+}(E)$ or $s_{-}(E)$ to obtain the continuum eigenfunction corresponding to energy $E$. [Here note that the hypergeometric function can be considered for complex arguments also]. For given $E$, this does not yield two independent eigenfunctions, but just one since $\Psi_{s_{+}(E), l}^{\gamma}(\theta, \phi)=\Psi_{s_{-}(E), l}^{\gamma}(\theta, \phi)$. Further, one can check explicitly that this function is everywhere regular for any integer $l$. [The secondorder differential equation for $F_{s, l}^{\gamma}(\theta)$ has another independent solution for given $E$, but it does not meet the regularity requirement near $\theta=0$ ]. Hence we have the desired continuum eigenfunction with energy $E>\frac{|R|}{4 m}\left(|\gamma|-\frac{1}{2}\right)^{2}$ represented by $\Psi_{s_{ \pm}(E), l}^{\gamma}(\theta, \phi)(l=0, \pm 1, \pm 2, \cdots)$, 
with $s_{ \pm}(E)$ specified by (4.42). This finding agrees with the result given in Ref.[26] (after making appropriate changes related to notational differences).

We remark that, in this case also, the energy eigenfunctions may be expressed using the complex coordinates $z, \bar{z}$ which are related to the above hyperbolic coordinates by

$$
z=\left(\tanh \frac{\theta}{2}\right) e^{i \phi}, \bar{z}=\left(\tanh \frac{\theta}{2}\right) e^{-i \phi} .
$$

Now, for operators $\pi_{\gamma}, \bar{\pi}_{\gamma}$, we have the following forms:

$$
\begin{aligned}
\pi_{\gamma} & =-\frac{i}{\sqrt{2} r}\left\{(1-z \bar{z}) \partial_{z}-|\gamma| \bar{z}\right\}=-\frac{i}{\sqrt{2} r}(1-z \bar{z})^{-|\gamma|+1} \vec{\partial}_{z}(1-z \bar{z})^{|\gamma|} \\
\bar{\pi}_{\gamma} & =-\frac{i}{\sqrt{2} r}\left\{(1-z \bar{z}) \partial_{\bar{z}}+|\gamma| z\right\}=-\frac{i}{\sqrt{2} r}(1-z \bar{z})^{|\gamma|+1} \vec{\partial}_{\bar{z}}(1-z \bar{z})^{-|\gamma|}
\end{aligned}
$$

Then, going through the steps parallel to the above development, we have the discrete energy eigenfunctions for instance described by

$$
\Psi_{s, l}^{\gamma}(z, \bar{z})=\sqrt{\frac{(2|\gamma|-2 s-1) \Gamma(2|\gamma|+l-s)}{4 \pi \Gamma(s+1) \Gamma(l+s+1) \Gamma(2|\gamma|-s)}}(1-z \bar{z})^{-|\gamma|}\left[(1-z \bar{z})^{2} \frac{\partial}{\partial z}\right]^{s} z^{l+s}(1-z \bar{z})^{2(|\gamma|-s)} .
$$

Using the associated Jacobi function, the last expression becomes (note that $\cosh \theta=\frac{1+z \bar{z}}{1-z \bar{z}}$ here)

$$
\Psi_{s, l}^{\gamma}(z, \bar{z})=\sqrt{\frac{(2|\gamma|-2 s-1) \Gamma(s+1) \Gamma(2|\gamma|+l-s)}{4 \pi \Gamma(l+s+1) \Gamma(2|\gamma|-s)}} z^{l}(1-z \bar{z})^{|\gamma|} \tilde{P}_{s}^{(l,-2|\gamma|-l)}\left(\frac{1+z \bar{z}}{1-z \bar{z}}\right)
$$

and one may consider the analytic continuation of this form to obtain the continuum energy eigenfunctions as well.

\section{CONCLUSIONS}

We have presented a supersymmetry-based approach as a systematic method for studying dynamics of a charged particle on a curved surface in the presence of a perpendicular magnetic field. The $\mathrm{N}=2$ supersymmetric system can be given in two equivalent ways, i.e., in the form of the curved-space generalization of the usual Pauli Hamiltonian or by a matrix Hamiltonian involving a pair of scalar superpartner Hamiltonians defined on the curved 
surface. In the cases where the system possesses the shape-invariance property in addition, complete solutions to the corresponding quantum mechanical problems can be found with the help of the simple operator technique analogous to that used to solve the harmonic oscillator problem. Based on this idea, we have obtained the energy levels and complete energy eigenfunctions explicitly for two specially interesting cases — the Landau Hamiltonian on the two-sphere and that on the hyperbolic plane. Compared to the case of the two-sphere where only discrete levels exist, the Landau Hamiltonian on the hyperbolic plane has complications due to the existence of additional continuum states. We have obtained the eigenfunctions appropriate to such continuum states by considering a suitable analytic continuation with the expression for discrete states. We hope that our consideration has exposed the power of the supersymmetry-based approach in studying Landau Hamiltonians defined on curved surfaces.

\section{ACKNOWLEDGMENTS}

This work was supported in part by the BK 21 project of the Ministry of Education, Korea and the Korea Research Foundation Grant 2001-015-DP0085. 


\section{REFERENCES}

[1] L.Landau, Z.Phys. 64 (1930), 629 ; L.Landau and E.Lifschitz, "Quantum Mechanics:Nonrelativistic Theory", Pergamon, New York, 1977.

[2] See, for example, C.Kittel, "Introduction to Solid State Physics", John Wiley \& Sons, New York, 1986.

[3] K. von Klitzing, Rev.Mod.Phys. 58 (1986), 519; K. von Klitzing, G.Dorda and M.Popper, Phys.Rev.Lett. 45 (1980), 494.

[4] For a review, see S.Girvin and R.Prange, "The Quantum Hall Effect", Springer-Verlag, New York, 1990 ; M.Stone (ed.), "Quantum Hall Effect", World Scientific Publ. Co., Singapore, 1992.

[5] R.Laughlin, Phys.Rev.Lett. 50 (1983) , 1395 ; Phys.Rev. B29 (1983), 3383.

[6] F.D.M.Haldane, Phys.Rev.Lett. 51 (1983), 605.

[7] M. de Crombrugghe and V.Rittenberg, Ann.Phys.(N.Y.)151 (1983), 99 ; A.Barducci, R.Casalbuoni and L.Lusanna, Nuovo Cimento 35A (1976), 377.

[8] R.J.Hughes, V.A.Kostelecký and M.M.Nieto, Phys.Rev. D34 (1986), 1100 ; V.A.Kostelecký, V.I.Man'ko, M.M.Nieto and D.R.Truax, Phys.Rev. A48 (1993), 951.

[9] Y.Aharonov and A.Casher, Phys.Rev. A19 (1979), 2461 ; R.Jackiw, Phys.Rev. D29 (1984), 2375.

[10] T.E.Clark, S.T.Love and S.Nowling, Mod.Phys.Lett. A15 (2000), 2105.

[11] L.Gendenshtein, Pis'ma Zh.Eksp.Teor.Fiz. 38 (1983), 299 [JETP Lett. 38 (1983), 356] ; R.Dutt, A.Khare and U.Sukhatme, Phys.Lett B181 (1986), 295.

[12] G.Junker, "Supersymmetric Methods in Quantum and Statistical Physics", SpringerVerlag, Berlin, 1996. 
[13] A.Comtet, Ann.Phys.(N.Y.) 173 (1987), 185 ; C.Grosche, Ann.Phys.(N.Y.) 187 (1988), 110.

[14] G.Dunne, Ann.Phys.(N.Y.) 215 (1992), 233.

[15] A.Pnueli, J.Phys.A:Math.Gen. 27 (1994), 1345 ; R.Iengo and D.Li, Nucl.Phys. 413 (1994), 735 .

[16] G.A.Mezincescu and L.Mezincescu, hep-th/0109002.

[17] See, for example, T.Eguchi, P.B.Gilkey and A.C.Hanson, Phys.Rep. 66 (1980), 213.

[18] F.A.Berezin and M.S.Marinov, JETP Lett. 21 (1975), 30 ; Ann.Phys.(N.Y.) 104 (1977), 336 ; R.Casalbuoni, Nuovo Cimento A33 (1976), 389.

[19] A.C.Davis, A.J.Macfarlane, P.C.Popat and J.W.van Holten, J.Phys. A17 (1984), 2945; A.J.Macfarlane and P.C.Popat, J.Phys. A17 (1984), 2955; V.de Alfaro, S.Fubini, G.Furlan and M.Roncadelli, Nucl.Phys. B296(1988), 402; Phys.Lett. B200 (1988), 323.

[20] L.Alvarez-Gaumé, Comm.Math.Phys. 90 (1983), 161; J.Phys. A16 (1983), 4177.

[21] V.de Alfaro, S.Fubini, G.Furlan and M.Roncadelli, Ref.19.

[22] P.A.M.Dirac, Proc.R.Soc. London A133 (1931), 60.

[23] M.Abramowitz and I.Stegun, "Handbook of Mathematical Functions," Dover, New York, 1972; N.Vilenkin, "Special Functions and the Theory of Group Representations," Amer.Math.Soc., Providence, RI, 1968.

[24] See, for instance, B.A.Dubrovin, A.T.Fomenko and S.P.Novikov, "Modern Geometry Methods and Applications", Part 1 (Springer-Verlag, 1984), Chap.2.

[25] A.Khare and U.P.Sukhatme, J.Phys. A21 (1988), L501.

[26] C.Grosche, Ann.Phys.(N.Y.) 201 (1990), 258. 